\title{
MutL homolog 1 contributes to temozolomide-induced autophagy via ataxia-telangiectasia mutated in glioma
}

\author{
YUHUI ZOU ${ }^{1,2}$, QIONG WANG ${ }^{3}$ and WEIMIN WANG ${ }^{1}$ \\ ${ }^{1}$ Department of Neurosurgery, Guangzhou General Hospital of Guangzhou Military Command, Guangzhou, \\ Guangdong 510010; ${ }^{2}$ Department of Neurosurgery, People's Liberation Army No.425 Hospital, Sanya, Hainan 572000; \\ ${ }^{3}$ Department of Medical Information, Guangzhou General Hospital of Guangzhou Military Command, \\ Guangzhou, Guangdong 510010, P.R. China
}

Received April 16, 2014; Accepted December 2, 2014

DOI: $10.3892 / \mathrm{mmr} .2015 .3293$

\begin{abstract}
In the present study, mutL homolog 1 (MLH1) small interfering (si)RNA, KU-55933, an ataxia-telangiectasia mutated (ATM) inhibitor, and compound $\mathrm{C}$, an adenosine monophosphate-activated protein kinase (AMPK) inhibitor, were used to investigate the mechanisms underlying temozolomide (TMZ)-induced autophagy and to determine the role of MLH1 and ATM in autophagy. MLH1 siRNA and KU-55933 inhibited the phosphorylation of AMPK and ULK1 and reduced the levels of autophagy. MLH1 siRNA inhibited the phosphorylation of ATM and attenuated TMZ cytotoxicity, whereas the inhibition of ATM-AMPK augmented TMZ cytotoxicity in inherently TMZ-sensitive glioma cells. Therefore, TMZ induced autophagy via the ATM-AMPK pathways and the activation of ATM-AMPK was MLH1-dependent. The inhibition of ATM-AMPK enhanced TMZ cytotoxicity in inherently TMZ-sensitive glioma cells.
\end{abstract}

\section{Introduction}

Previous studies have demonstrated that $\mathrm{O}^{6}$-methylguanine DNA methyltransferase (MGMT) can eliminate the temozolomide (TMZ)-induced $\mathrm{O}^{6}$-methylguanine $\left(\mathrm{O}^{6} \mathrm{MeG}\right)$ DNA adduct and leads to TMZ resistance (1-3). However, the MGMT promoter is methylated in almost $50 \%$ of glioblastoma specimens and the methylation status of the MGMT promoter

Correspondence to: Professor Weimin Wang, Department of Neurosurgery, Guangzhou General Hospital of Guangzhou Military Command, 111 Liuhua Road, Guangzhou, Guangdong 510010, P.R. China

E-mail: gzwangwm0@163.com

Abbreviations: TMZ, temozolomide; MMR, DNA mismatch repair; MLH1, mutL homolog 1; ATM, ataxia-telangiectasia mutated; AMPK, adenosine monophosphate-activated protein kinase; ULK1, unc-51-like autophagy activating kinase 1

Key words: autophagy, mutL homolog 1, ataxia-telangiectasia mutated, glioma, temozolomide in the primary tumor is retained at recurrence $(4,5)$. This suggests other mechanisms are involved in TMZ resistance, which remain to be elucidated.

TMZ-induced autophagy functions as a cytoprotective mechanism against TMZ toxicity and contributes to TMZ resistance. The inhibition of autophagy augments TMZ cytotoxicity in glioma (6-8). Following nutrient restriction or stress, adenosine monophosphate-activated protein kinase (AMPK) is involved in the formation of the autophagosome by interacting with unc-51-like autophagy activating kinase 1 (ULK1) and induces autophagy $(9,10)$. Ataxia-telangiectasia mutated (ATM), a serine/threonine protein kinase, leads to the phosphorylation of AMPK following ionizing radiation or nutrient restriction, which suggests that ATM is the upstream kinase of AMPK $(11,12)$. However, the role of ATM-AMPK in TMZ-induced autophagy remains to be elucidated.

DNA mismatch repair (MMR), a genetic repair pathway, is composed of MutS and MutL heterodimers $(3,5)$. The $\mathrm{O}^{6} \mathrm{MeG} / \mathrm{T}$ mismatches are immediately recognized by MutS (3) and the mutL homolog 1 (MLH1) MMR protein then migrates from the nucleus to the cytoplasm via nuclear export sequences, possibly signaling DNA damage to downstream pathways (13). However, whether MLH1, a DNA damage messenger, is involved in TMZ-induced autophagy remains to be elucidated.

The present study hypothesized that MLH1 signaled DNA damage to ATM or AMPK and induced autophagy in glioma following treatment with TMZ. To investigate this hypothesis, the activation of AMPK-ULK1 and the levels of autophagy following treatment with MLH1 small interfering (si)RNA and KU-55933 were assessed. The association between MLH1 and ATM was investigated and the effects of the inhibition of autophagy, by knock down of MLH1 and inhibition of ATM-AMPK, on the cytotoxicity of TMZ were evaluated.

\section{Materials and methods}

Cell culture and reagents. The U87MG, U251 and SHG-44 glioma cell lines were obtained from the Cell Bank of Chinese Academy of Sciences (Shanghai, China). All cells were plated at a density of $1 \times 10^{6}$ cells/well in a 6 -well plate and maintained in Dulbecco's modified Eagle's medium 
(Gibco Life Technologies, Carlsbad, CA, USA) supplemented with $10 \%$ fetal bovine serum (PAA Laboratories, Pasching, Austria) at $37^{\circ} \mathrm{C}$ in a $5 \% \mathrm{CO}_{2}$-humidified atmosphere. The U87MG cells were cultured and passaged in the presence of increasing concentrations of TMZ $(30,60,90,120,150,180$, 210, 240, 270 and $300 \mu \mathrm{M})$ to generate TMZ-resistant cell lines, termed U87TMZ. The glioma cells were divided into the following four groups: i) control; ii) TMZ $(100 \mu \mathrm{M}, 72 \mathrm{~h})$; iii) KU-55933 (10 $\mu \mathrm{M}, 72 \mathrm{~h}) /$ compound $\mathrm{C}(5 \mu \mathrm{M})$; iv) TMZ $(100 \mu \mathrm{M}, 72 \mathrm{~h})+\mathrm{KU}-55933(10 \mu \mathrm{M}, 72 \mathrm{~h}) /$ compound $\mathrm{C}(5 \mu \mathrm{M}$, $72 \mathrm{~h})$ The TMZ was supplied by Tasly Pharmaceutical Co., Ltd. (Tianjin, China). KU-55933 (cat. no. sc-202963) and compound C (cat. no. P5499) were purchased from Santa Cruz Biotechnology, Inc. (Dallas, TX, USA) and Sigma-Aldrich (St. Louis, MO, USA), respectively. The TMZ (100 $\mu \mathrm{M})$, KU-55933 $(10 \mu \mathrm{M})$ and compound $\mathrm{C}(5 \mu \mathrm{M})$ were dissolved in dimethylsulfoxide (DMSO; Sigma-Aldrich). The final concentration of DMSO in the culture medium did not exceed $0.01 \%$, and therefore did not effect cell viability or protein expression.

siRNA transfection. For siRNA transfection, the MLH1, 5'-G CCAUGUGGCUCAUGUUACdTdT-3' siRNA oligo was used and 5'-GCUCAGAUCAAUACGGAGAdTdT-3' was used as a non-specific negative control. The siRNAs were purchased from Genepharma Co., Ltd. (Shanghai, China). The cells were transfected with $100 \mathrm{nM}$ siRNA using Lipofectamine RNAiMAX transfection reagent (Invitrogen Life Technologies, Carlsbad, CA, USA). At $48 \mathrm{~h}$ post-transfection, the DMEM culture media was refreshed and the cells were exposed to $100 \mu \mathrm{M} \mathrm{TMZ}$ ro $72 \mathrm{~h}$, followed by western blot analysis or the detection of autophagosomes. Cells without siRNA transfection were used as a negative control.

Western blot analysis. Briefly, cells were dissolved and boiled in Laemmli buffer (Beyotime Institute of Biotechnology, Shanghai, China) for $5 \mathrm{~min}$, separated with $10 \%$ SDS-PAGE (Beyotime Institute of Biotechnology) and transferred to polyvinylidene fluoride membranes (Bio-Rad Laboratories, Inc., Hercules, CA, USA) at $80 \mathrm{~mA}$ for $2 \mathrm{~h}$. Following blocking with bovine serum albumin (Thermo Fisher Scientific, Waltham, MA, USA), the membranes were incubated with the primary antibodies in Tris-buffered saline with Tween-20 (TBST; Amresco LLC, Solon, OH, USA) at $37^{\circ} \mathrm{C}$ for $2 \mathrm{~h}$. Subsequently, secondary antibodies in TBST were added and incubated for $2 \mathrm{~h}$ at room temperature. Bound secondary antibodies were detected using an enhanced chemiluminescence detection system (Beyotime Institute of Biotechnology). Antibodies specific for MLH1 (cat. no. 3515), phosphorylated (p)ATM (Ser1981; cat. no. 5883), total ATM (cat. no. 2873), total AMPK $\alpha$ (cat. no. 2532), pAMPK $\alpha$ (Thr172; cat. no. 2535), pULK1 (Ser467; cat. no. 4634) and microtubule-associated protein 1 light chain $3 \beta$ (LC3B; cat. no. 2775), with $\beta$-actin (cat. no. 4970) as a loading control, were purchased from Cell Signaling Technology, Inc (Danvers, MA, USA). Polyclonal anti- $\gamma \mathrm{H} 2 \mathrm{AX}$ antibody (cat. no. ab2893) was obtained from Abcam (Cambridge, MA, USA). Histone subtypes $(\gamma \mathrm{H} 2 \mathrm{AX})$ provide a sensitive marker of DNA double-strand breaks (DSBs) (14) and LC3B, which is cleaved into LC3B-I and LC3B-II during autophagy, was used as an autophagy marker $(15,16)$.
Detection of the autophagosome/acidic vesicular organelles (AVOs). Following TMZ and/or KU-55933/Compound C treatment, acridine orange (cat. no. sc-358795, Santa Cruz Biotechnology, Inc.) was added at a final concentration of $1 \mu \mathrm{g} / \mathrm{ml}$ for $15 \mathrm{~min}$. The cells were collected, excited (488 nm) and analyzed by flow cytometric analysis using a Guava Easycyte 5HT flow cytometer (EMD Millipore, Billerica, MA, USA). The nucleoli of the acridine orange-stained cells fluoresced bright green and the acidic vesicles emitted a bright red fluorescence. The cells containing AVOs were identified as double positive cells.

Detection of apoptosis. The apoptotic cells were detected using an annexin V-fluorescein isothiocyanate (FITC)/propidium iodide (PI) apoptosis detection kit (cat. no. C1063; Beyotime Institute of Biotechnology, Jiangsu, China) according to the manufacturer's instructions, using flow cytometric analysis on a Guava Easycyte 5HT flow cytometer (EMD Millipore). The data were analyzed using Guava Soft 2.2 software (EMD Millipore).

Cell viability analysis. MTT assays were performed to assess the sensitivity of the cells to the drug treatments. Briefly, the cells were seeded at a density of 3,000 cells/well in 96-well microplates. Following drug treatment, $20 \mu \mathrm{l}$ MTT $(5 \mathrm{mg} / \mathrm{ml})$ was added to each well and the plates were incubated at $37^{\circ} \mathrm{C}$ for $4 \mathrm{~h}$. The culture media was subsequently replaced with $100 \mu 1$ DMSO to lyse the cells, followed by the measurement of absorbance at $570 \mathrm{~nm}$ using a Thermo Fisher Scientific Multiskan GO microplate reader (Thermo Fisher Scientific, Inc., Waltham, MA, USA).

Statistical analysis. All the experiments were performed in triplicate and data are expressed as the mean \pm standard deviation. Statistical analysis of the data was performed using Student's t-test for two groups or a one-way analysis of variance for three or more groups. $\mathrm{P}<0.05$ was considered to indicate a statistically significant difference.

\section{Results}

TMZ-induced autophagy is MLHI-dependent. Western blot analysis revealed that TMZ failed to induce the phosphorylation of AMPK or ULK1 following MLH1 knock down in the U87MG, U251 or SHG-44 cells (Fig. 1A and B). In the TMZ+MLH1 siRNA group, the cleavage of LC3B was decreased and the number of AVOs was reduced, including under cytotoxic stress, compared with the TMZ group $(10.85 \pm 1.36 \%$, vs. $34.02 \pm 1.77 \%$, respectively; $\mathrm{P}<0.001)$. Additionally,theMLH1 siRNAgrouphadfewerAVOscompared with the control group $(5.60 \pm 0.64 \%$, vs. $12.29 \pm 0.91 \%$, respectively; P<0.001; Fig. 1C), consistent with the LC3B cleavage results (Fig. 1B). This suggested that MLH1 was important in the activation of TMZ-induced autophagy.

TMZ induces autophagy via PATM, which is MLH1-dependent. TMZ failed to induce the phosphorylation of AMPK or ULK1 when used in combination with $10 \mu \mathrm{M}$ KU-55933. The TMZ+KU-55933 group had lower expression levels of LC3B-I and LC3B-II compared with the TMZ or the control group 


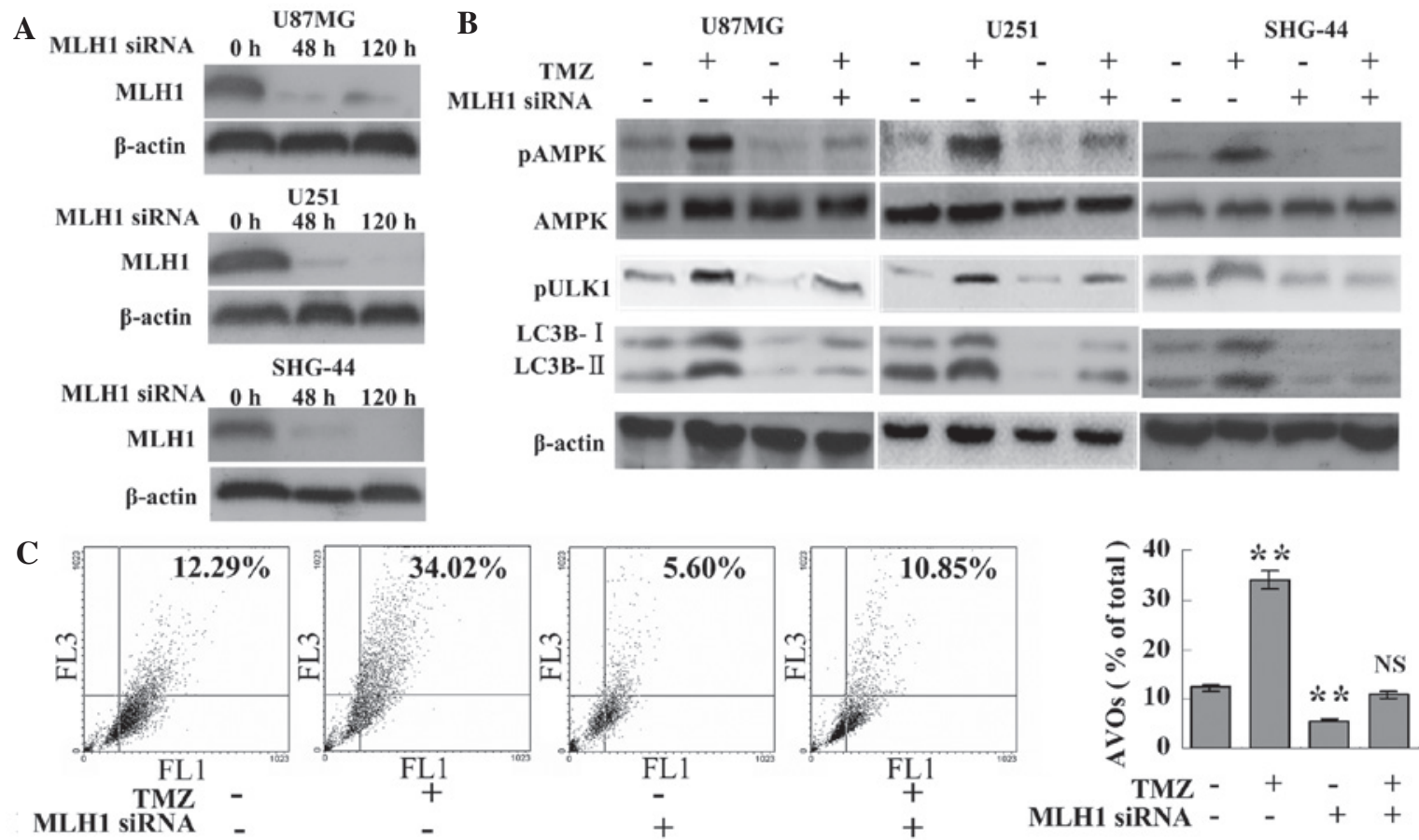

Figure 1. TMZ induces autophagy via MLH1. (A) Glioma cells were transfected with $100 \mathrm{nM}$ MLH1 siRNA and harvested at the indicated time points for western blot analysis. (B) Glioma cells and the MLH1-knockdown glioma cells (100 nM MLH1 siRNA for $48 \mathrm{~h}$ ) were incubated with $100 \mu \mathrm{M}$ TMZ or vehicle (DMSO) for $72 \mathrm{~h}$. Following treatment, the cells were collected for western blot analysis. (C) U87MG cells and the MLH1-knockdown U87MG cells were incubated with $100 \mu \mathrm{M} \mathrm{TMZ}$ or vehicle for $72 \mathrm{~h}$. Following treatment, the cells were collected for AVO detection using flow cytometric analysis. The data are expressed as the mean \pm standard deviation ${ }^{* *} \mathrm{P}<0.01$, vs. control group; $\mathrm{n}=3$ for each group). NS, not statistically significant compared with the control group; MLH1, mutL homolog 1; TMZ, temozolomide; siRNA, small interfering RNA; AMPK, adenosine monophosphate-activated protein kinase; ULK, unc-51-like autophagy activating kinase 1; LC3B, microtubule-associated protein 1 light chain $3 \beta$; AVO, acidic vesicular organelle, p, phosphorylated; FL1, green fluorescence; FL3, red fluorescence.

A
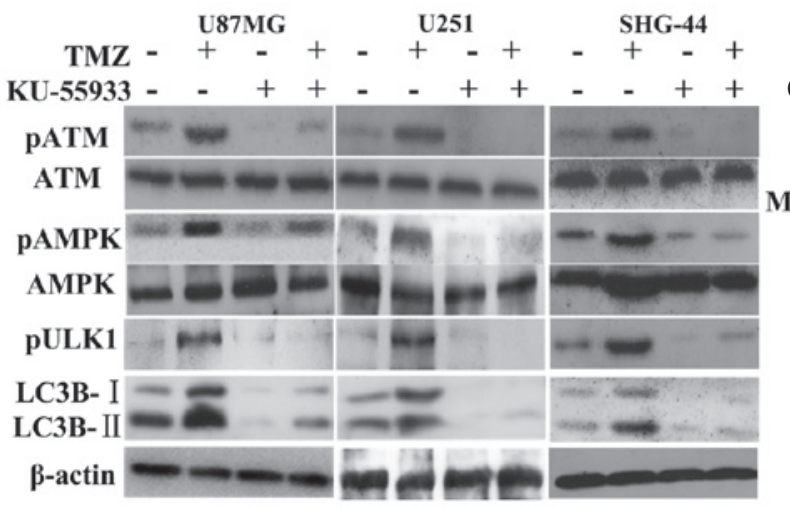

C

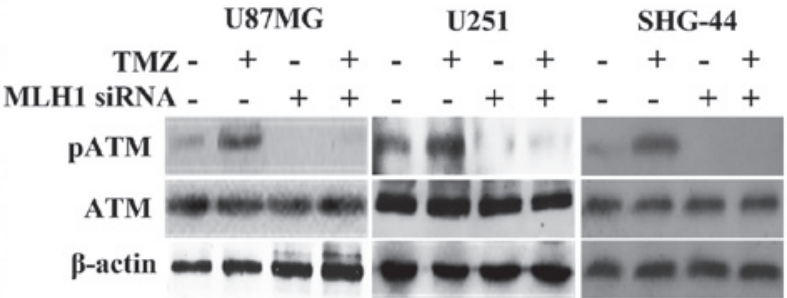

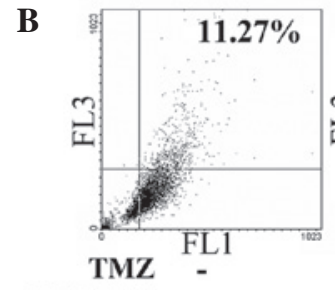

KU-55933 -

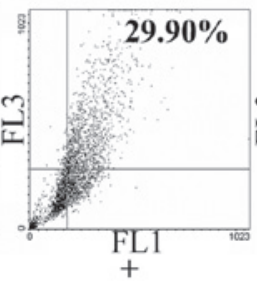

-
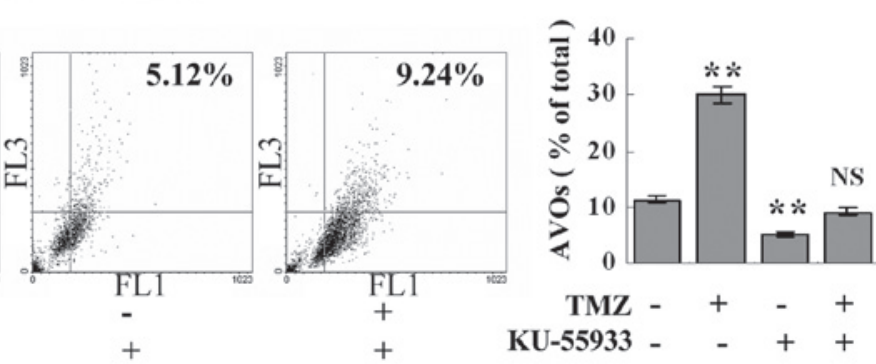

Figure 2. TMZ induces autophagy via pATM, which is MLH1-dependent. (A) U87MG, U251 and SHG-44 cells were treated with $100 \mu \mathrm{M}$ TMZ and/or $10 \mu \mathrm{M} \mathrm{KU}-55933$, as indicated, for $72 \mathrm{~h}$. Subsequently, the cells were collected for western blot analysis. dimethyl sulfoxide was used as a negative control. (B) U87MG cells were treated with $100 \mu \mathrm{M} \mathrm{TMZ}$ and/or $10 \mu \mathrm{M} \mathrm{KU}-55933$, as indicated, for $72 \mathrm{~h}$. Following treatments, the cells were collected and the number of AVOs were detected using flow cytometric analysis. (C) Glioma cells and MLH1-knockdown glioma cells (100 $\mu \mathrm{M}$ MLH1 siRNA for $48 \mathrm{~h}$ ) were incubated with $100 \mu \mathrm{M}$ TMZ or vehicle (DMSO) for $72 \mathrm{~h}$. Subsequently, the levels of pATM were assessed using western blot analysis. The data are expressed as the mean \pm standard deviation ( ${ }^{* *} \mathrm{P}<0.01$, vs. control group; $\mathrm{n}=3$ for each group). NS, not statistically significant compared with the control group; MLH1, mutL homolog 1; ATM, ataxia telangiectsia; p, phosphorylated; TMZ, temozolomide; siRNA, small interfering RNA; AMPK, adenosine monophosphate-activated protein kinase; ULK, unc-51-like autophagy activating kinase 1; LC3B, microtubule-associated protein 1 light chain $3 \beta$; AVO, acidic vesicular organelle; FL1, green fluorescence; FL3, red fluorescence. 

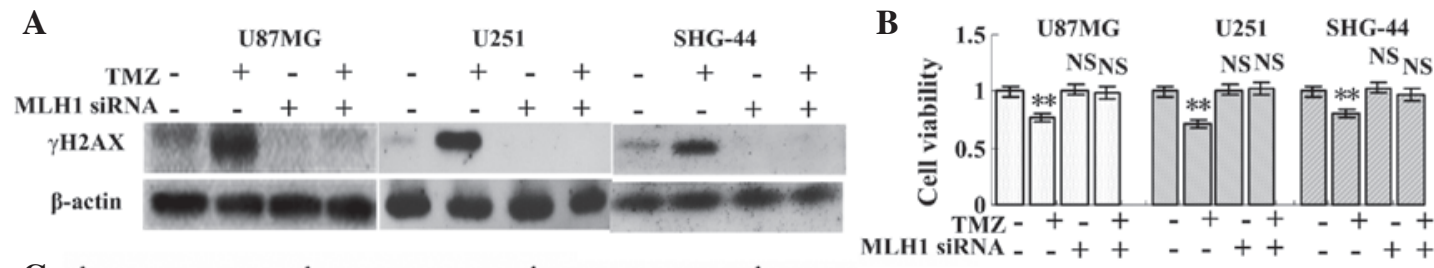

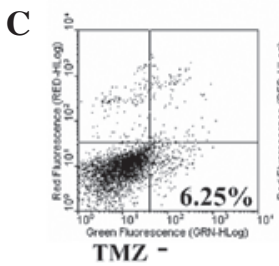

MLH1 SiRNA -

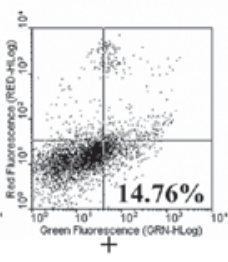

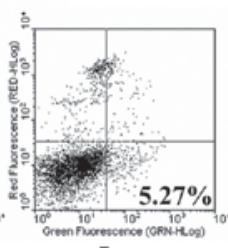

$+$ D

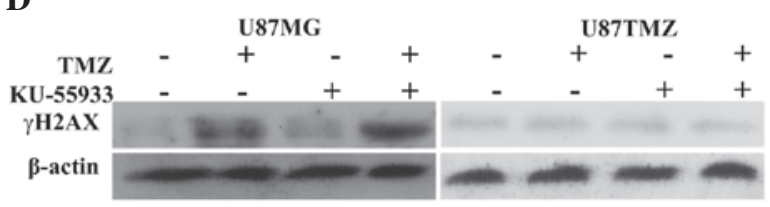

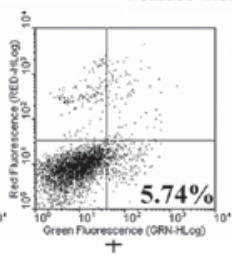

$+$

E

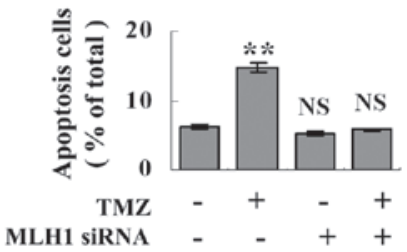

U U87MG U87TMZ

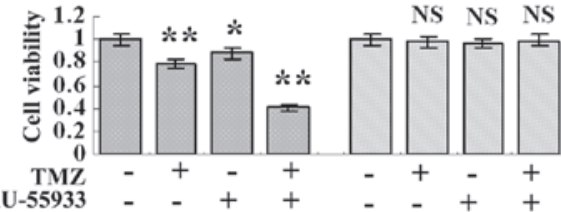

Figure 3. Knock down of MLH1 results in TMZ resistance, whereas inhibition of ATM augments TMZ cytotoxicity in inherently TMZ-sensitive cells (A) Glioma cells and MLH1-knockdown glioma cells (100 $\mu \mathrm{M}$ MLH1 siRNA for $48 \mathrm{~h}$ ) were incubated with $100 \mu \mathrm{M}$ TMZ or vehicle for $72 \mathrm{~h}$. The cells were then collected to detect the expression of $\gamma \mathrm{H} 2 \mathrm{AX}$ by western blot analysis. (B) Glioma cells and MLH1-knockdown glioma cells were seeded at a density of 3,000 cells/well in 96-well microplates and treated with $100 \mu \mathrm{M}$ TMZ or vehicle (DMSO) for $72 \mathrm{~h}$. MTT assays were performed to assess the cell viability (n=6 for each group). (C) U87MG cells were treated as in (A) and apoptosis was detected by annexin-V-fluorescin isothiocyanate/propidium iodide double staining ( $\mathrm{n}=3$ for each group). (D and E) U87MG and U87TMZ cells were treated with $100 \mu \mathrm{M} \mathrm{TMZ}$ and/or $10 \mu \mathrm{M} \mathrm{KU}-55933$, as indicated, for 72 h. (D) Western blot analysis was then performed to detect the expression of $\gamma \mathrm{H} 2 \mathrm{AX}$. Dimethyl sulfoxide was used as a negative control. (E) MTT assays were performed to assess the cell viability ( $\mathrm{n}=6$ for each group). The data are expressed as the mean \pm standard deviation ( $\mathrm{P}<0.05$ and ${ }^{* *} \mathrm{P}<0.01$, vs. control group). NS, not statistically significant; MLH1, mutL homolog 1; TMZ, temozolomide; siRNA, small interfering RNA.

(Fig. 2A). Consistently, the inhibition of ATM resulted in fewer AVOs in the TMZ+KU-55933 group compared with the TMZ group $(9.24 \pm 0.38 \%$, vs. $29.90 \pm 2.14 \%$, respectively; $\mathrm{P}<0.001$; Fig. 2B). The levels of LC3B cleavage and AVOs were also reduced in the KU-55933 group compared with the control group (Fig. 2A and B). Therefore, TMZ-induced autophagy and the phosphorylation of AMPK were ATM-dependent. Subsequently, the association between MLH1 and ATM was investigated. Compared with the significant phosphorylation of ATM in the TMZ group, the knock down of MLH1 inhibited the phosphorylation of ATM in the MLH1 siRNA and the TMZ+MLH1 siRNA groups (Fig. 2C), which indicated that the TMZ-induced phosphorylation of ATM was MLH1-dependent.

Knock down of MLH1 leads to TMZ resistance. Since autophagy is critical for tumor survival, the effect of MLH1 knock down on TMZ cytotoxicity was evaluated. The knock down of MLH1 resulted in a significant decrease in the expression of $\gamma \mathrm{H} 2 \mathrm{AX}$ (Fig. 3A). The knock down of MLH1 also favored U87MG, U251 and SHG-44 cell growth following treatment with TMZ. The MLH1 siRNA and TMZ+MLH1 siRNA groups exhibited normal cell growth, while the cells in the TMZ group exhibited the slowest rate of cell growth (Fig. 3B). Additionally, the levels of apoptosis in the MLH1 siRNA and TMZ+MLH1 siRNA groups were $5.27 \pm 0.67 \%$ and $5.74 \pm 1.24 \%$, respectively, which were not significantly different compared with the control group $(6.25 \pm 1.15 \%)$, however, levels were lower compared with the TMZ group (14.76 $\pm 1.59 \%$; Fig. 3C). This indicated that knock down of MLH1 resulted in TMZ resistance, despite inhibition of autophagy.

ATM inhibition augments TMZ cytotoxicity in inherently TMZ-sensitive glioma cells. In the TMZ+KU-55933 group, increased expression of $\gamma \mathrm{H} 2 \mathrm{AX}$ and reduced cell viability were observed compared with the other groups in the U87MG cells. By contrast, TMZ and KU-55933 had no effect on the expression of $\gamma \mathrm{H} 2 \mathrm{AX}$ or on the viability of the U87TMZ cells, suggesting that the inhibition of ATM augmented TMZ cytotoxicity in the inherently TMZ-sensitive cells (Fig. 3D and E).

AMPK inhibition disrupts autophagy and augments TMZ cytotoxicity in inherently TMZ-sensitive glioma cells. The inhibition of AMPK resulted in lower expression levels of LC3B-I and LC3B-II in the TMZ+compound C group compared with the TMZ group, suggesting that the inhibition of AMPK interrupted TMZ-induced autophagy (Fig. 4A and C). Since autophagy favors cell survival, the present study investigated whether the inhibition of AMPK augmented TMZ cytotoxicity. As expected, compound C augmented TMZ cytotoxicity, which was indicated by an increase in the expression of $\gamma \mathrm{H} 2 \mathrm{AX}$ and in the number of apoptotic cells in the TMZ+compound $\mathrm{C}$ group compared with the other groups (Fig. 4C and D). The cell viability analysis also revealed that the slowest rate of cell growth was observed in the TMZ+compound $\mathrm{C}$ group (Fig. 4B).

In the U87TMZ cells, the expression levels of pULK1 and LC3B cleavage in the TMZ group were indistinguishable from those in the control group, suggesting that TMZ failed 


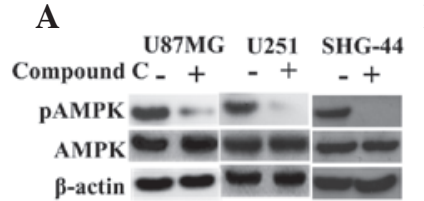

B 1

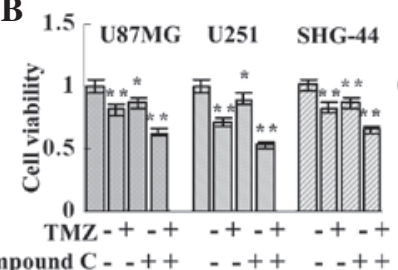

D
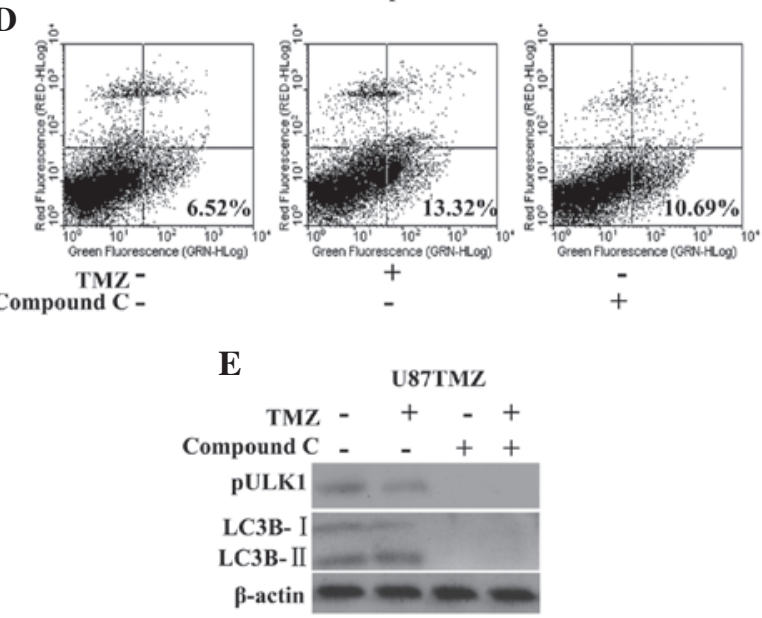

C U87MG Compound C - $-++-c_{-}+-++$ LC3B-I $=-1+=-$

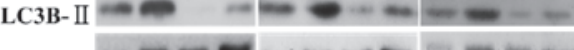
$\gamma \mathbf{H} 2 \mathrm{AX}$

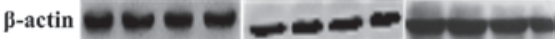
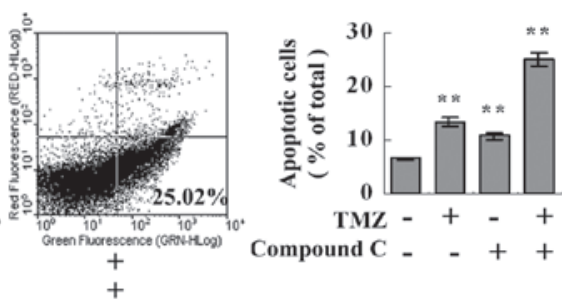$$
\text { U87TMZ }
$$

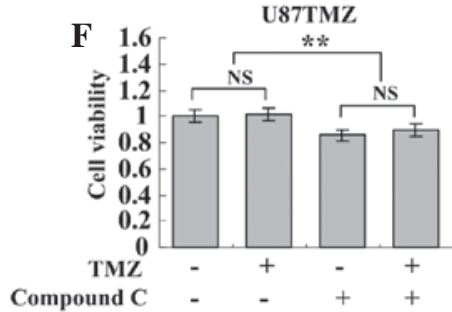

Figure 4. AMPK inhibition decreases levels of autophagy and augments TMZ cytotoxicity in inherently TMZ-sensitive glioma cells. (A) U87MG, U251 and SHG-44 cells were treated with $5 \mu \mathrm{M}$ compound C or vehicle (DMSO) for $72 \mathrm{~h}$. The cells were then harvested for western blot analysis to determine the levels of pAMPK $\underline{\alpha}$ (Thr172). (B-D) U87MG, U251 and SHG-44 cells were treated with $100 \mu \mathrm{M}$ TMZ and/or $5 \mu \mathrm{M}$ compound C for $72 \mathrm{~h}$. (B) MTT assays were performed to assess the cell viability ( $\mathrm{n}=6$ for each group) and (C) western blot analysis was performed to detect the expression of LC3B and $₹ \mathrm{H} 2 \mathrm{AX}$. (D) $\mathrm{U} 87 \mathrm{MG}$ cells were treated with $100 \mu \mathrm{M}$ TMZ and/or $5 \mu \mathrm{M}$ compound $\mathrm{C}$ for $72 \mathrm{~h}$. Apoptosis was detected using annexin-V-fluorescin isothiocyanate/propidium iodide double staining ( $\mathrm{n}=3$ for each group). U87TMZ cells were treated with $100 \mu \mathrm{M}$ TMZ and/or $5 \mu \mathrm{Mm}$ compound C for $72 \mathrm{~h}$. (E) Western blot analysis was performed to detect the expression levels of pULK1 and LC3B. (F) MTT assays were performed to assess the cell viability (n=6 for each group). The data are expressed as the mean \pm standard deviation $\left({ }^{*} \mathrm{P}<0.05\right.$ and ${ }^{* *} \mathrm{P}<0.01$, vs. control group or indicated group). NS, not statistically significant, MLH1, mutL homolog 1; TMZ, temozolomide; siRNA, small interfering RNA; AMPK, adenosine monophosphate-activated protein kinase; ULK, unc-51-like autophagy activating kinase 1; LC3B, microtubule-associated protein 1 light chain $3 \beta$; p, phosphorylated.

to increase the levels of autophagy in the TMZ-resistant glioma cells. Although compound $\mathrm{C}$ decreased LC3B cleavage and reduced cell viability in the compound $\mathrm{C}$ and the $\mathrm{TMZ}+$ compound $\mathrm{C}$ groups, no significant differences were observed in the levels of pULK1, LC3B cleavage or cell viability between these two groups, suggesting that the reduction in autophagy in the U87TMZ cells was not associated with TMZ (Fig. 4E and F). These results indicated that a reduction in autophagy using AMPK inhibitors only augmented TMZ cytotoxicity in inherently TMZ-sensitive tumor lines.

\section{Discussion}

Following 6-thioguanine or 5-fluorouracil treatment, MLH1 is involved in $\mathrm{G} 2 / \mathrm{M}$ cell cycle arrest for damage repair and, at a later stage, in the induction of autophagy for irreparable DNA damage (17-19). The present study indicated that MLH1 is also important in TMZ-induced autophagy.

AMPK is a conserved sensor of intracellular energy, which is activated in response to low nutrient availability and cellular stresses and is involved in the initiation of autophagosome formation by interacting with the ULK1 autophagy-initiating kinase $(9,10)$. Following DNA damage or nutrient restriction, pATM leads to the phosphorylation of AMPK, indicating that ATM is an upstream kinase of AMPK $(11,12)$. The results from the present study demonstrated that TMZ-induced AMPK phosphorylation was regulated by ATM. Following
ATM inhibition, TMZ failed to induce the phosphorylation of AMPK or ULK1, which led to decreased levels of LC3B cleavage and AVO formation. The activation of AMPK-ULK1 was also under MLH1 regulation, however the association between MLH1 and ATM remained to be determined.

Following the detection of DNA mismatches by MutS, MLH1 is recruited to the foci, undergoes conformational changes through cycles of ATP binding and hydrolysis and recruits and/or activates downstream effector proteins (20-22). The present study hypothesized that ATM was a downstream protein of MLH1 and the results supported this hypothesis, as TMZ induced autophagy via the phosphorylation of ATM in an MLH1-dependent manner.

Notably, knock down of MLH1 and inhibition of ATM-AMPK decreased autophagy, however, the opposite effect was observed on TMZ cytotoxicity. TMZ-induced $\mathrm{O}^{6} \mathrm{MeG} / \mathrm{T}$ mismatches are firstly bound and recognized by MutS, which subsequently recruits MutL to the foci $(21,23)$. The MutS-MutL complex excises the mispaired thymine, while $\mathrm{O}^{6} \mathrm{MeG}$ persists in the template strand (3). Such failed replication cycles with the repeated reinsertion and excision of thymine results in replication fork collapse and DSBs (3). MLH1 is a major component of the MMR pathway and MLH1 deficiency leads to failure of the MMR pathway to recognize the $\mathrm{O}^{6} \mathrm{MeG} / \mathrm{T}$ mismatches, leading to TMZ resistance $(3,24,25)$. By contrast, ATM-AMPK had no effect on the failed cell cycles or on the formation of DSB, however, it was involved 
in autophagy. Therefore, the inhibition of ATM-AMPK decreased autophagy and increased the therapeutic efficacy of TMZ in the inherently TMZ-sensitive cells. Kanzawa et al demonstrated that inhibiting autophagy at an early stage using 3-methyladenine suppresses the characteristic recruitment of LC3 to autophagosome membranes and rescues tumor cells from cell death (26). Whether MLH1 is involved in the localization of LC3 requires further investigation.

The present study demonstrated for the first time, to the best of our knowledge, that TMZ induced autophagy through the ATM-AMPK pathways, which occured in an MLH1-dependent manner. Although the knock down of MLH1 and inhibition of ATM-AMPK decreased TMZ-induced autophagy, MLH1 knock down led to TMZ-resistance in the glioma cells, while ATM-AMPK inhibition augmented TMZ cytotoxicity.

\section{Acknowledgements}

This study was supported by the National Natural Science Foundation of China (no. 81302201), the China Postdoctoral Science Foundation (no. 2012M512182) and the Guangdong Natural Science Foundation (no. S2012040006588).

\section{References}

1. Cen L, Carlson BL, Pokorny JL, et al: Efficacy of protracted temozolomide dosing is limited in MGMT unmethylated GBM xenograft models. Neuro Oncol 15: 735-746, 2013.

2. Jiang G, Li LT, Xin Y, Zhang L, Liu YQ and Zheng JN: Strategies to improve the killing of tumors using temozolomide: targeting the DNA repair protein MGMT. Curr Med Chem 19: 3886-3892, 2012.

3. Zhang J, Stevens MF and Bradshaw TD: Temozolomide: Mechanisms of action, repair and resistance. Curr Mol Pharmacol 5: 102-114, 2012.

4. Skiriute D, Vaitkiene P, Saferis V, et al: MGMT, GATA6, CD81, DR4, and CASP8 gene promoter methylation in glioblastoma. BMC Cancer 12: 218, 2012.

5. Felsberg J, Thon N, Eigenbrod S, et al: Promoter methylation and expression of MGMT and the DNA mismatch repair genes MLH1, MSH2, MSH6 and PMS2 in paired primary and recurrent glioblastomas. Int J Cancer 129: 659-670, 2011.

6. Lin CJ, Lee CC, Shih YL, et al: Resveratrol enhances the therapeutic effect of temozolomide against malignant glioma in vitro and in vivo by inhibiting autophagy. Free Radic Biol Med 52: 377-391, 2012.

7. Katayama M, Kawaguchi T, Berger MS and Pieper RO: DNA damaging agent-induced autophagy produces a cytoprotective adenosine triphosphate surge in malignant glioma cells. Cell Death Differ 14: 548-558, 2007.

8. Natsumeda M, Aoki H, Miyahara H, et al: Induction of autophagy in temozolomide treated malignant gliomas. Neuropathology 31 486-493, 2011.
9. Wong PM, Puente C, Ganley IG and Jiang X: The ULK1 complex: sensing nutrient signals for autophagy activation. Autophagy 9 : 124-137, 2013.

10. She C, Zhu LQ, Zhen YF, Wang XD and Dong QR: Activation of AMPK protects against hydrogen peroxide-induced osteoblast apoptosis through autophagy induction and NADPH maintenance: New implications for osteonecrosis treatment? Cell Signal 26: 1-8, 2014.

11. Singh K, Matsuyama S, Drazba JA and Almasan A: Autophagy-dependent senescence in response to DNA damage and chronic apoptotic stress. Autophagy 8: 236-251, 2012.

12. Duan X, Ponomareva L, Veeranki S and Choubey D: IFI16 induction by glucose restriction in human fibroblasts contributes to autophagy through activation of the ATM/AMPK/p53 pathway. PLoS One 6: e19532, 2011.

13. Brieger A, Adam R, Passmann S, Plotz G, Zeuzem S and Trojan J: A CRM1-dependent nuclear export pathway is involved in the regulation of MutLalpha subcellular localization. Genes Chromosomes Cancer 50: 59-70, 2011.

14. Takahashi A and Ohnishi T: Does gammaH2AX foci formation depend on the presence of DNA double strand breaks? Cancer Lett 229: 171-179, 2005.

15. Murrow L and Debnath J: Autophagy as a stress-response and quality-control mechanism: Implications for cell injury and human disease. Annu Rev Pathol 8: 105-137, 2013.

16. Wu J, Dang Y, Su W, et al: Molecular cloning and characterization of rat LC3A and LC3B - two novel markers of autophagosome. Biochem Biophys Res Commun 339: 437-442, 2006.

17. Zeng X and Kinsella TJ: BNIP3 is essential for mediating 6-thioguanine- and 5-fluorouracil-induced autophagy following DNA mismatch repair processing. Cell Res 20: 665-675, 2010.

18. Zeng X and Kinsella TJ: Mammalian target of rapamycin and S6 kinase 1 positively regulate 6-thioguanine-induced autophagy. Cancer Res 68: 2384-2390, 2008

19. Zeng X and Kinsella TJ: A novel role for DNA mismatch repair and the autophagic processing of chemotherapy drugs in human tumor cells. Autophagy 3: 368-370, 2007.

20. Johnson JR, Erdeniz N, Nguyen M, Dudley S and Liskay RM: Conservation of functional asymmetry in the mammalian MutLalpha ATPase. DNA Repair (Amst) 9: 1209-1213, 2010.

21. Polosina YY and Cupples CG: MutL: conducting the cell's response to mismatched and misaligned DNA. Bioessays 32: $51-59,2010$.

22. Chahwan R, van Oers JM, Avdievich E, et al: The ATPase activity of MLH1 is required to orchestrate DNA double-strand breaks and end processing during class switch recombination. J Exp Med 209: 671-678, 2012.

23. Quiros S, Roos WP and Kaina B: Processing of O6-methylguanine into DNA double-strand breaks requires two rounds of replication whereas apoptosis is also induced in subsequent cell cycles. Cell Cycle 9: 168-178, 2010.

24. Sarkaria JN, Kitange GJ, James CD, et al: Mechanisms of chemoresistance to alkylating agents in malignant glioma. Clin Cancer Res 14: 2900-2908, 2008.

25. Taverna P, Liu L, Hanson AJ, Monks A and Gerson SL: Characterization of MLH1 and MSH2 DNA mismatch repair proteins in cell lines of the NCI anticancer drug screen. Cancer Chemother Pharmacol 46: 507-516, 2000.

26. Kanzawa T, Germano IM, Komata T, Ito H, Kondo Y and Kondo S: Role of autophagy in temozolomide-induced cytotoxicity for malignant glioma cells. Cell Death Differ 11: 448-457, 2004. 